\title{
Mortality After Discontinuation of Primary Care-Based Chronic Opioid Therapy for Pain: a Retrospective Cohort Study
}

\author{
Jocelyn R. James, MD', JoAnna M. Scott, $P h D^{2}$, Jared W. Klein, MD, MPH', \\ Sara Jackson, $M D, M P H^{7}$, Christy McKinney, $P h D, M P H^{3}$, Matthew Novack, $M S^{3}$, \\ Lisa Chew, MD, MPH', and Joseph O. Merrill, MD, MPH
}

'Department of Medicine, Division of General Internal Medicine, Harbonview Medical Center, University of Washington School of Medicine, Seattle, WA, USA; ${ }^{2}$ University of Missouri - Kansas City School of Dentistry, Kansas City, MO, USA; ${ }^{3}$ Tacoma Family Medicine, Multicare, Tacoma, WA, USA.

BACKGROUND: Despite known risks of using chronic opioid therapy (COT) for pain, the risks of discontinuation of COT are largely uncharacterized.

OBJECTIVE: To evaluate mortality, prescription opioid use, and primary care utilization of patients discontinued from COT, compared with patients maintained on opioids. DESIGN: Retrospective cohort study of patients with chronic pain enrolled in an opioid registry as of May 2010. PARTICIPANTS: Patients with chronic pain enrolled in the opioid registry of a primary care clinic at an urban safety-net hospital in Seattle, WA.

MAIN OUTCOMES AND MEASURES: Discontinuation from the opioid registry was the exposure of interest. Pre-specified main outcomes included mortality, prescription and primary care utilization data, and reasons for discontinuation. Data was collected through March 2015.

KEY RESULTS: The study cohort comprised 572 patients with a mean age of $54.9 \pm 10.1$ years. COT was discontinued in 344 patients (60.1\%); 254 (73.8\%) discontinued patients subsequently filled at least one opioid prescription in Washington State, and 187 (54.4\%) continued to visit the clinic. During the study period, $119(20.8 \%)$ registry patients died, and 21 (3.7\%) died of definite or possible overdose: 17 (4.9\%) discontinued patients died of overdose, whereas 4 (1.75\%) retained patients died of overdose. Most patients had at least one provider-initiated reason for COT discontinuation. Discontinuation of COT was associated with a hazard ratio for death of 1.35 (95\% CI, 0.92 to 1.98, $p=0.122)$ and for overdose death of 2.94 (1.01-8.61, $p=0.049)$, after adjusting for age and race.

CONCLUSIONS: In this cohort of patients prescribed COT for chronic pain, mortality was high. Discontinuation of

Prior Presentations Study data on reasons for discontinuation were presented at the Western Student and Resident Medical Research Forum (February 2016). Key results of this study were presented in abstract and poster form at the Societu of General Internal Medicine (Avril 2018).

Electronic supplementary material The online version of this article (https://doi.org/10.1007/s11606-019-05301-2) contains supplementary material. which is available to authorized users.

Received September 27, 2018

Revised April 24, 2019

Accepted July 25, 2019

Published online August 29, 2019
COT did not reduce risk of death and was associated with increased risk of overdose death. Improved clinical strategies, including multimodal pain management and treatment of opioid use disorder, may be needed for this highrisk group.

KEY WORDS: chronic pain; primary care; addiction.

$\mathrm{J}$ Gen Intern Med 34(12):2749-55

DOI: $10.1007 / \mathrm{s} 11606-019-05301-2$

(c) Society of General Internal Medicine 2019

\section{INTRODUCTION}

Between 2000 and 2010, use of opioids for non-malignant pain rose by $73 \%$ in the USA. ${ }^{1}$ During a comparable period, there was a tripling of age-adjusted rates of deaths that involved prescription-type natural or semisynthetic opioids with or without heroin. While most overdose deaths involve more than one substance, and death rate data do not document whether the opioid identified was associated with an actual prescription, ${ }^{2}$ it is likely that widespread increases in opioid prescribing since the turn of the century contributed to the increase in opioid related deaths. ${ }^{3}$ Increased recognition of this epidemic of opioid misuse and overdose, combined with awareness of the limited evidence of long-term benefit of opioids for chronic pain, ${ }^{4}$ led to efforts to reduce harms associated with opioid prescribing. Changes in opioid prescribing policies have taken many forms, including guidelines on risk assessment, opioid dosing, and monitoring; patient agreements; and use of statewide electronic prescription drug monitoring programs (PDMPs). ${ }^{5-7}$ Yet despite reductions in dispensed opioid prescriptions and evidence of reduced diversion of opioids, ${ }^{6}$ prescription opioid-related deaths in the USA increased from 2014 to 2016 and accounted for about $40 \%$ of opioid-related deaths in 2016. ${ }^{8,9}$

It is likely that guidelines for safer prescribing will help limit the number of people newly exposed to the harms of opioids. An area of less certainty, however, is how patients already on chronic opioid therapy (COT) for pain, generally defined as regular opioid prescriptions for at least 3 months, have been affected by changes in prescribing and monitoring 
policies. Since increased scrutiny and stricter clinic policies will lead some patients to leave or transition care, voluntarily or otherwise, we undertook this study to understand why patients are discontinued from COT and what happens to them afterward.

\section{MATERIALS AND METHODS}

\section{Design Overview}

We retrospectively examined the characteristics and clinical outcomes of a cohort of patients with chronic pain who received chronic opioids managed through a primary care clinic based opioid registry from 2010 to 2015 , a period of substantial change in prescribing practices at the clinic. We examined reasons for discontinuation from COT, and we evaluated use of prescription opioids and continuity of primary care after discontinuation. We also compared mortality and overdose mortality of patients whose COT was discontinued with those of patients who were maintained on COT.

\section{Study Setting}

The study was performed within a large academic primary care clinic based at Harborview Medical Center in Seattle, WA, a safety-net hospital. The clinic serves approximately 4500 patients from diverse backgrounds in 20,000 visits annually. In early 2010 , the clinic implemented a comprehensive effort to improve the safety of opioid prescribing for chronic pain; this included guidelines on risk assessment and monitoring (including urine screens), dosing recommendations, and the creation of an interdisciplinary panel to set clinic policies and provide management recommendations. A ceiling dose of $120 \mathrm{mg}$ morphine equivalents per day (MED) was recommended and providers were encouraged to taper patients on higher doses. Once Washington State's PDMP became fully available in January 2012, PDMP checks became routine and were entered into the electronic health record (EHR).

The clinic maintains a pharmacist-managed "opioid registry," which serves as the organizational tool for opioid prescribing policies and procedures. Use of the registry for patients on COT is an explicit expectation of providers and is incentivized by pharmacist assistance managing refills. Discontinuing a patient from the registry involves communication between pharmacists and providers. It would be unusual and impractical for a provider to discontinue the patient from the registry while intending to continue regular opioid prescribing, making registry discharge an excellent proxy for a provider's attempt to discontinue COT.

\section{Study Participants}

The study cohort consisted of clinic patients 18 years and older who were prescribed COT for chronic pain and enrolled in the opioid registry as of May 2010. Patients were excluded if they were prescribed opioids but not enrolled in the registry or if they joined the registry after May 2010. Patient data was collected from May 1, 2010, through March 31, 2015. Clinic policies did not specifically address opioid prescribing for patients with active malignancies; thus, these patients could be included on the registry.

\section{Data Sources}

Study data sources included the EHR, death records from the Washington State Department of Health, and clinic-based opioid registry files. Washington State death files were obtained through March 2015. Demographics, clinical data, and utilization data were electronically abstracted from the EHR and merged with death records using unique identifiers. Opioid registry files included Microsoft Excel spreadsheets managed by clinic pharmacists and Washington State PDMP data; the PDMP was queried for all registry patients as part of a quality improvement effort in 2015 and the results were added to the patient's clinic opioid registry records. The Washington State PDMP documents all scheduled medication prescriptions filled throughout the state; its use was mandatory for non-Veterans Affairs pharmacies by January 2012.

Dates and reasons for discontinuation of COT and prescription data were abstracted through chart review of the EHR and clinic opioid registry files and imported into a study database in REDCap.

\section{Definition of COT}

Our primary exposure of interest was discontinuation of COT, represented by discontinuation from the opioid registry. Patients who were on the clinic opioid registry as of May 1, 2010, and remained on the registry through March 2015, or who died while on the registry, were considered to have continued COT. Those who were removed from the registry at any point during the study period for any reason other than death were considered to have had COT discontinued. We did not determine COT duration for each patient, as registry data did not include COT initiation dates. The date of discontinuation was often directly recorded in opioid registry files or in telephone or visit notes in the EHR; if not, it was calculated by adding days' supply to the date of the last continuous monthly prescription by the prescribing provider, using PDMP data. If the date was unavailable based on these methods (PDMP data was not available prior to 2012), it was approximated as the midway point between the dates at which the patient was present and absent from the opioid registry files. Because versions of these files were saved on a roughly monthly basis, these estimated dates would differ minimally from the actual dates. For patients who died within 30 days of the date of discontinuation, additional chart review was performed to determine whether death occurred prior to or following discontinuation. 
The small number of patients who had COT discontinued but later returned to the registry were still categorized as having had COT discontinued, as their primary care provider had made a decision to discontinue opioids at least once.

\section{Measures}

To assess characteristics of our study cohort, we abstracted demographic information (age at baseline, sex, race/ethnicity, language, marital status, and insurance status); substance use, mental health, and other medical diagnoses; and prescription of controlled medications. Diagnoses were abstracted from all available EHR data. Baseline opioid dose was determined by examining opioid registry Excel files for the period prior to discontinuation and identifying the first of three consecutive opioid prescriptions written by the same prescriber for the same opioid medication, dose, and number of tablets. It was converted to morphine equivalent dose (MED) ${ }^{10}$ and summed, in the case of multiple prescribed opioids.

For the discontinued group, we characterized subsequent opioid prescriptions according to PDMP records. Patients were not reclassified with regard to opioid registry status based on retrospective review of PDMP records. To assess continuity of primary care, clinic and hospital visit data were electronically abstracted from the EHR. All patients continued in the registry were assumed to have completed regular primary care visits, as this was required for ongoing receipt of COT.

All-cause mortality and death due to overdose were assessed for all patients. Death by overdose was characterized as definite or possible based on a priori classification of information provided to the Washington State Department of Health by the medical examiner or other certifying physician. Death due to definite overdose was assigned when death records specified drug overdose or intoxication; death due to possible overdose was assigned to deaths due to respiratory failure (Supplement). A composite of definite and possible overdose was used in the analysis of overdose-related death.

\section{Characterization of Reasons for Discontinuation}

In many cases, a reason for discontinuation was noted explicitly in opioid registry files; in other cases, contributing reasons appeared in provider and pharmacist notes. Multiple reasons for COT discontinuation could be recorded. Abstraction of reasons for COT discontinuation was performed by one co-author (MN); several co-authors ( $\mathrm{SJ}$, $\mathrm{JJ}, \mathrm{JK}, \mathrm{JM}$ ) independently reviewed $10 \%$ of patients' records and found $>95 \%$ concordance. Specific reasons for COT discontinuation were listed, grouped (MN, JK), and analyzed through an iterative process to identify key patterns. Reasons deemed similar from a clinical perspective were combined. Because each participant could have multiple reasons for discontinuation listed, the total number of unique individuals in each category was determined. Reasons that were most common and/or most clinically significant were selected for inclusion in the results.

\section{Statistical Analyses}

Descriptive statistics were calculated for variables of interest. Frequencies were calculated for each category of reasons for COT discontinuation. Cox proportional hazard models adjusting for age and race were used to determine associations between discontinuation of COT and all-cause mortality and between discontinuation of COT and death due to overdose. Fisher's exact test was used to determine associations between reasons for discontinuation of COT and overdose death. The significance level was set to 0.05 . All analysis was performed in Stata 14.2 (StataCorp LP, College Station, TX).

\section{Institutional Review}

The study was approved by the Institutional Review Board at the University of Washington.

\section{RESULTS}

\section{Baseline Characteristics}

The study cohort comprised 572 patients on COT for chronic pain managed through the clinic opioid registry. The mean age of study patients was $54.9 \pm 10.1$ years; $261(45.6 \%)$ were women, 299 (52.3\%) were white, 206 $(36.0 \%)$ were black, and $489(85.5 \%)$ had public insurance. Substance use and mental health disorders were common (Table 1).

At the study start date in 2010, 145 (25.3\%) of patients were prescribed a total morphine equivalent dose (MED) between 120 and $359 \mathrm{mg} /$ day and $61(10.7 \%)$ were prescribed at least $360 \mathrm{mg} /$ day. Three hundred eighty-seven $(67.7 \%)$ patients were prescribed short-acting opioids, 169 (29.5\%) were prescribed long-acting morphine or oxycodone, and 162 (28.3\%) were prescribed methadone (Table 1).

\section{Reasons for COT Discontinuation}

COT was discontinued in $344(60.1 \%)$ patients during the study period. Reasons for discontinuation were categorized as patient-initiated or provider-initiated (Table 2). Common patient-initiated reasons for discontinuation included moving or transferring care, while provider-initiated reasons for discontinuation largely involved behavioral concerns and safety issues. Most patients $(77 \%)$ had at least one provider-initiated reason for COT discontinuation, whereas $18 \%$ had only patient-initiated reasons; the reason for discontinuation was unclear in $5 \%$ of patients.

\section{Mortality and Overdose}

Among the entire study cohort, 119 patients (20.8\%) died during the study period and $21(3.7 \%)$ died of overdose, corresponding to death rates per 100 personyears of 4.73 (95\% CI 3.95-5.66) and 0.83 (95\% CI $0.54-1.28)$, respectively. The hazard ratio for death from 
Table 1 Characteristics of Patients on Chronic Opioid Therapy, by Retention in Registry Versus Discontinuation of Opioids between 2010 and 2015

\begin{tabular}{|c|c|c|c|}
\hline & Total $(N=572)$ & Retained $(N=228)$ & COT Discontinued $(N=344)$ \\
\hline \multicolumn{4}{|l|}{ Demographic characteristics } \\
\hline \multicolumn{4}{|l|}{ Age in years } \\
\hline $18-39$ & $43(7.5 \%)$ & $13(5.7 \%)$ & $30(8.7 \%)$ \\
\hline $40-49$ & $111(19.4 \%)$ & $35(15.4 \%)$ & $76(22.1 \%)$ \\
\hline $50-59$ & $262(45.8 \%)$ & $114(50.0 \%)$ & $148(43.0 \%)$ \\
\hline$>59$ & $156(27.3 \%)$ & $66(28.9 \%)$ & $90(26.2 \%)$ \\
\hline Female & $261(45.6 \%)$ & $110(48.2 \%)$ & $151(43.9 \%)$ \\
\hline \multicolumn{4}{|l|}{ Race } \\
\hline White/Caucasian & $299(52.3 \%)$ & $119(52.2 \%)$ & $180(52.3 \%)$ \\
\hline Black or African American & $206(36 \%)$ & $76(33.3 \%)$ & $130(37.8 \%)$ \\
\hline Asian & $9(1.6 \%)$ & $7(3.1 \%)$ & $2(0.6 \%)$ \\
\hline Native American or Alaska Native & $18(3.1 \%)$ & $11(4.8 \%)$ & $7(2 \%)$ \\
\hline Other & $32(5.6 \%)$ & $11(4.8 \%)$ & $21(6.1 \%)$ \\
\hline Missing & $8(1.4 \%)$ & $4(1.8 \%)$ & $4(1.2 \%)$ \\
\hline English speaking & $559(97.7 \%)$ & $225(98.7 \%)$ & $334(97.1 \%)$ \\
\hline \multicolumn{4}{|l|}{ Marital status } \\
\hline Single & $293(51.2 \%)$ & $116(50.9 \%)$ & $177(51.5 \%)$ \\
\hline Married or domestic partner & $104(18.2 \%)$ & $37(16.2 \%)$ & $67(19.5 \%)$ \\
\hline Divorced or separated & $142(24.8 \%)$ & $63(27.6 \%)$ & $79(23.0 \%)$ \\
\hline Widowed & $32(5.6 \%)$ & $12(5.3 \%)$ & $20(5.8 \%)$ \\
\hline Other or missing & $1(0.2 \%)$ & $0(0 \%)$ & $1(0.3 \%)$ \\
\hline \multicolumn{4}{|l|}{ Insurance status } \\
\hline Self-pay or charity & $43(7.5 \%)$ & $13(5.7 \%)$ & $30(8.7 \%)$ \\
\hline Medicare/Medicaid & $489(85.5 \%)$ & $196(86 \%)$ & $293(85.2 \%)$ \\
\hline Private insurance & $31(5.4 \%)$ & $16(7 \%)$ & $15(4.4 \%)$ \\
\hline Other & $9(1.6 \%)$ & $3(1.3 \%)$ & $6(1.7 \%)$ \\
\hline \multicolumn{4}{|l|}{ Health characteristics } \\
\hline Current tobacco use & $225(39.3 \%)$ & $98(43.0 \%)$ & $127(36.9 \%)$ \\
\hline Alcohol use disorder & $110(19.2 \%)$ & $42(18.4 \%)$ & $68(19.8 \%)$ \\
\hline History of other substance use & $197(34.4 \%)$ & $65(28.5 \%)$ & $132(38.4 \%)$ \\
\hline Mental health disorders & $420(73.4 \%)$ & $173(75.9 \%)$ & $247(71.8 \%)$ \\
\hline Depression & $373(65.2 \%)$ & $162(71.1 \%)$ & $211(61.3 \%)$ \\
\hline Anxiety disorder & $221(38.6 \%)$ & $88(38.6 \%)$ & $133(38.7 \%)$ \\
\hline PTSD & $72(12.6 \%)$ & $28(12.3 \%)$ & $44(12.8 \%)$ \\
\hline Schizophrenia or psychotic disorder & $76(13.3 \%)$ & $25(11 \%)$ & $51(14.8 \%)$ \\
\hline Mania and bipolar disorders & $37(6.5 \%)$ & $9(3.9 \%)$ & $28(8.1 \%)$ \\
\hline \multicolumn{4}{|l|}{ Chronic medical conditions } \\
\hline Hypertension & $435(76 \%)$ & $182(79.8 \%)$ & $253(73.5 \%)$ \\
\hline Diabetes & $206(36 \%)$ & $91(39.9 \%)$ & $115(33.4 \%)$ \\
\hline COPD & $148(25.9 \%)$ & $63(27.6 \%)$ & $85(24.7 \%)$ \\
\hline Sleep apnea & $153(26.7 \%)$ & $77(33.8 \%)$ & $76(22.1 \%)$ \\
\hline Hepatitis C infection & $144(25.2 \%)$ & $52(22.8 \%)$ & $92(26.7 \%)$ \\
\hline Congestive heart failure & $90(15.7 \%)$ & $43(18.9 \%)$ & $47(13.7 \%)$ \\
\hline \multicolumn{4}{|l|}{ Body mass index } \\
\hline Underweight $\left(<18.5 \mathrm{~kg} / \mathrm{m}^{2}\right)$ & $15(2.6 \%)$ & $4(1.8 \%)$ & $11(3.2 \%)$ \\
\hline Healthy weight $\left(18.5-24.9 \mathrm{~kg} / \mathrm{m}^{2}\right)$ & $108(18.9 \%)$ & $48(21.1 \%)$ & $60(17.4 \%)$ \\
\hline Overweight $\left(25-29.9 \mathrm{~kg} / \mathrm{m}^{2}\right)$ & $136(23.8 \%)$ & $54(23.7 \%)$ & $82(23.8 \%)$ \\
\hline Obese $\left(30.0+\mathrm{kg} / \mathrm{m}^{2}\right)$ & $249(43.5 \%)$ & $115(50.4 \%)$ & $134(39 \%)$ \\
\hline Missing & $64(11.2 \%)$ & $7(3.1 \%)$ & $57(16.6 \%)$ \\
\hline \multicolumn{4}{|l|}{ Opioid characteristics } \\
\hline Opioid indication ${ }^{*}$ & $553(96.7 \%)$ & $224(98.2 \%)$ & $329(95.6 \%)$ \\
\hline Back/spine pain & $454(79.4 \%)$ & $188(82.5 \%)$ & $266(77.3 \%)$ \\
\hline Pain in extremity & $471(82.3 \%)$ & $198(86.8 \%)$ & $273(79.4 \%)$ \\
\hline Abdominal pain & $199(34.8 \%)$ & $86(37.7 \%)$ & $113(32.8 \%)$ \\
\hline Chronic wounds & $82(14.3 \%)$ & $33(14.5 \%)$ & $49(14.2 \%)$ \\
\hline Neuropathic pain or headache & $206(36 \%)$ & $88(38.6 \%)$ & $118(34.3 \%)$ \\
\hline \multicolumn{4}{|l|}{ Opioid dose in mg/day MED } \\
\hline$<30$ & $88(15.4 \%)$ & $31(13.6 \%)$ & $57(16.6 \%)$ \\
\hline $30-59$ & $100(17.5 \%)$ & $43(18.9 \%)$ & $57(16.6 \%)$ \\
\hline $60-119$ & $98(17.1 \%)$ & $51(22.4 \%)$ & $47(13.7 \%)$ \\
\hline $120-359$ & $145(25.3 \%)$ & $79(34.6 \%)$ & $66(19.2 \%)$ \\
\hline$\geq 360$ & $61(10.7 \%)$ & $21(9.2 \%)$ & $40(11.6 \%)$ \\
\hline Missing & $80(14 \%)$ & $3(1.3 \%)$ & $77(22.4 \%)$ \\
\hline Any short-acting natural or semisynthetic opioids** & $387(67.7 \%)$ & $186(81.6 \%)$ & $201(58.4 \%)$ \\
\hline Any long-acting natural or semisynthetic opioids** & $169(29.5 \%)$ & $91(39.9 \%)$ & $78(22.7 \%)$ \\
\hline Any methadone & $162(28.3 \%)$ & $71(31.1 \%)$ & $91(26.5 \%)$ \\
\hline Any fentanyl patch & $5(0.9 \%)$ & $3(1.3 \%)$ & $2(0.6 \%)$ \\
\hline
\end{tabular}

*In many cases, more than one pain condition was associated with COT. **For this cohort, this includes all opioids except for the synthetic opioids methadone and fentanyl 
Table 2 Reasons for Discontinuation of COT $(n=344)$

\begin{tabular}{|c|c|c|}
\hline & Patients & Percent \\
\hline \multicolumn{3}{|l|}{ Patient initiated } \\
\hline Moved & 20 & 5.8 \\
\hline Incarcerated & 4 & 1.2 \\
\hline \multicolumn{3}{|l|}{ Transferred care } \\
\hline Another PCP & 29 & 8.4 \\
\hline Nursing home palliative care, hospice & 28 & 8.1 \\
\hline Pain/addiction specialist & 18 & 5.2 \\
\hline Other/unspecified & 6 & 1.7 \\
\hline Other & 15 & 4.4 \\
\hline \multicolumn{3}{|l|}{ Provider initiated } \\
\hline \multicolumn{3}{|l|}{ Urine toxicology abnormality } \\
\hline Unexpected positive & 85 & 24.7 \\
\hline Unexpected negative & 37 & 10.8 \\
\hline Declined or altered urine specimen & 9 & 2.6 \\
\hline \multicolumn{3}{|l|}{ Behavioral issues } \\
\hline Missed appointments & 62 & 18.0 \\
\hline Multi-sourcing & 31 & 9.0 \\
\hline Dosing disagreements & 30 & 8.7 \\
\hline Perceived lack of engagement in care & 27 & 7.8 \\
\hline Aggressive, threatening, or violent behavior & 23 & 6.7 \\
\hline Early refill requests & 18 & 5.2 \\
\hline Perceived opioid seeking & 17 & 4.9 \\
\hline Illicit use of prescription opioids & 15 & 4.4 \\
\hline Lost/stolen medications and/or abnormal pill & 11 & 3.2 \\
\hline count & & \\
\hline \multicolumn{3}{|l|}{ Other safety concerns } \\
\hline Past or present substance use disorder & 51 & 14.8 \\
\hline Mental health condition & 48 & 14.0 \\
\hline Medical condition & 35 & 10.2 \\
\hline Limited pain response and/or lack of & 33 & 9.6 \\
\hline \multicolumn{3}{|l|}{ functional improvement } \\
\hline Impaired respiratory function & 21 & 6.1 \\
\hline Alcohol use disorder & 18 & 5.2 \\
\hline Suicidality & 9 & 2.6 \\
\hline Concurrent benzodiazepine use & 7 & 2.0 \\
\hline Medication side effect & 7 & 2.0 \\
\hline Non-opioid or unspecified overdose & 6 & 1.7 \\
\hline Opioid overdose & 5 & 1.5 \\
\hline Missing/unknown & 20 & 5.8 \\
\hline
\end{tabular}

Sums to more than $100 \%$ because subjects could have more than one reason for discontinuation

all causes among patients who discontinued COT in comparison with those patients maintained in the registry was 1.27 (95\% CI $0.87-1.86, p=0.219)$ in an unadjusted analysis and 1.35 (95\% CI $0.92-1.98, p=$ 0.122) after adjusting for age and race (Fig. 1).

Among overdose deaths, 17 were classified as definite overdose and 4 as possible overdose. Seventeen discontinued patients $(4.9 \%)$ died of overdose, whereas 4 retained patients $(1.75 \%)$ died of overdose. The hazard ratio for overdose death among patients who discontinued COT was 2.82 (95\% CI $0.95-8.41, p=0.062)$ in an unadjusted analysis and 2.94 (95\% CI 1.01-8.61, $p=0.049)$ after adjusting for age and race. All seventeen overdose deaths among patients who discontinued COT occurred in those who had at least one provider-initiated reason for discontinuation. While there was not a statistically significant association between reason(s) for discontinuation and overdose deaths, there was a trend toward higher death rates in patients with at least one provider-initiated reason for discontinuation compared with patients with only patient-initiated reasons for discontinuation $(6.4 \%$ vs $0 \%$; Fisher's exact $p=0.051)$.

\section{Controlled Substance Use Among Patients Discontinued from the Registry}

Among discontinued patients, 254 (73.8\%) filled an opioid prescription in Washington State after discontinuation and 183 (53.4\%) filled a prescription within the last year of the study period. Following COT discontinuation, short-acting opioid prescriptions were filled by $242(70.3 \%)$ patients and methadone prescriptions by 54 (15.7\%).

\section{Continuity of Primary Care Among Patients Discontinued from the Registry}

Among patients discontinued from the opioid registry, 187 (54.4\%) visited the clinic two or more times during the remainder of the study period and $228(66.3 \%)$ visited Harborview Medical Center at least twice.

\section{DISCUSSION}

In this retrospective cohort study of patients with chronic pain enrolled in the chronic opioid registry of a primary care clinic, nearly $21 \%$ of patients died during nearly 5 years of follow-up and $4 \%$ died from overdose. COT discontinuation, which occurred in more than half of opioid registry patients, appeared to be provider-initiated in at least $77 \%$ of patients. There was no statistically significant difference in all-cause mortality between maintained and discontinued patients. Overdose death was more common in those who discontinued COT; those with provider-initiated reasons for discontinuation appeared to be at increased risk, though this finding did not reach statistical significance. Ongoing prescription opioid use and disruption of primary care continuity were both common after discontinuation of COT. These data call into question the safety of opioid discontinuation as an effort to reduce the risks of COT.

The high rate of all-cause mortality in this study cohort likely reflects in part the high prevalence of chronic medical, psychiatric, and substance use conditions that are also associated with mortality, and in part the known risks of opioids, especially at high doses. ${ }^{11,12}$ In fact, drug and alcohol poisonings have contributed to the reversal of decades of declining midlife mortality. ${ }^{13}$

While some data suggests that a high percentage of patients on COT for chronic pain will agree to a voluntary taper, ${ }^{14}$ realworld experience suggests that tapering is often providerinitiated. Discontinuation of COT often occurs in response to "red flag" behaviors or safety concerns and generally represents an attempt to improve patient safety; liability concerns may also play a role. In this era of increased attention on opioid-associated harms, ${ }^{4}$ cautionary guidance regarding new initiation of opioids, ${ }^{15}$ and heightened scrutiny of prescribers, there is little known about the effects of COT discontinuation, ${ }^{16}$ minimal guidance regarding patients prescribed high-dose COT or demonstrating concerning behaviors, and an absence of support for patients whose providers implement 


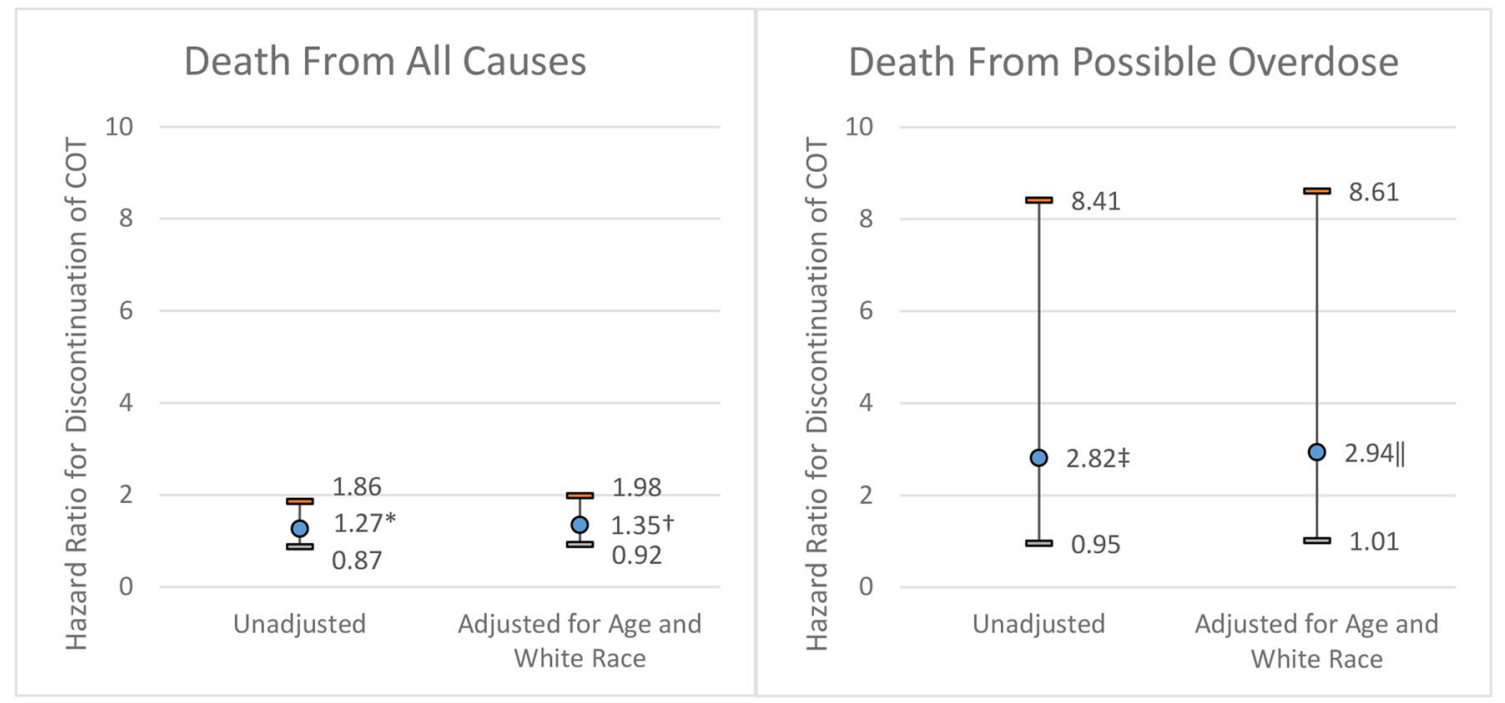

Figure 1 Risk of death from all causes and from possible or definite overdose, comparing patients whose opioids were discontinued with patients maintained on the clinic opioid registry. COT, chronic opioid therapy; see Supplement for definitions of possible or definite overdose; * $p=$ $0.219 ;{ }^{\dagger} p=0.122 ;{ }^{\dagger} p=0.062 ;{ }^{n} p=0.049$.

rapid taper schedules or cease prescribing altogether. Patients and providers have expressed concern that non-consensual tapering and discontinuation of COT risk destabilization and illicit opioid use, severe opioid withdrawal, worsening pain, loss of function, and/or suicidality. ${ }^{17-19}$

This study's finding that overdose death was increased in patients discontinued from COT could relate to interruption of other medical care, loss of tolerance, and/or destabilization of an underlying opioid use disorder. Estimates of risk of opioid use disorder in patients on COT vary widely, but recent data suggest the risk is dose related and higher than previously thought, up to 122 -fold for patients on very high doses. ${ }^{20}$ It is also possible that in this study, the high-risk behaviors that led to opioid discontinuation also contributed to risk of death, since its observational design limits conclusions about causality.

Opioid prescribing as identified by the PDMP was common after opioid discontinuation. High rates of continued opioid prescribing after an overdose event have also been documented, ${ }^{21}$ underscoring the difficulty of changing prescribing patterns in spite of clear risks. If opioid use disorder is at least in part driving concerns about continuing COT, it may be misguided to discontinue opioids without careful identification and adequate treatment of this disorder. Treatment with buprenorphine or referral to methadone maintenance programs may be our safest options when clinical monitoring of patients on COT results in safety concerns. Since the years studied here, our clinic has implemented a Nurse Care Manager model of office-based opioid treatment of opioid use disorders. ${ }^{22}$ While most patients treated with buprenorphine in this program are seeking care for opioid use disorder, some are patients whose opioid use disorder was diagnosed or emerged in the context of COT for chronic pain. Primary care providers who prescribe COT may benefit from obtaining a waiver and prescribing buprenorphine to their patients with chronic pain and opioid use disorder, potentially avoiding the disruption of primary care common in this cohort.

Another potential source of increased risk of overdose death in discontinued patients is poorly managed chronic pain. It is possible that comprehensive, multimodal pain management for patients with chronic pain on COT would also reduce risk.

Our study has several important limitations. Our use of a clinic registry rather than pharmacy records to define our population may have missed some COT patients who were prescribed opioids outside the registry. We are confident that clinic policies and protocols supporting use of the registry were effective and that opioids prescribed outside it were likely intermittent or low dose. This could bias our estimate of death rates, but should not bias our comparison of those retained or discontinued from COT. Similarly, opioid discontinuation was defined through registry and EHR review rather than pharmacy records, and inaccuracies in our review could explain the high level of subsequent opioid prescribing after COT discontinuation. Reasons for discontinuation were based on chart review rather than direct patient or provider survey, but for most patients, we found clear documentation of safety issues or violations of our clinic controlled substance agreement, increasing confidence that COT discontinuation was intended.

Our study has additional limitations related to its setting and methods. It was performed at a single site, and the relatively small sample size and retrospective design limit the results. Measures were imperfect: data from the EHR are often incomplete and subject to misclassification, and we lacked access to out-of-state prescription and death information, to PDMP information prior to January 2012, and to non-prescription opioid use. We also relied on death records to characterize cause of death, a potentially imprecise process. 


\section{CONCLUSIONS}

In conclusion, we found that primary care patients on COT had a high mortality risk and that discontinuation of COT was not associated with a reduced risk of death and in fact was associated with an increased risk of death from overdose. While more research is needed to evaluate the generalizability of these findings, and to understand how to better protect patients on COT from harm, caution when discontinuing COT is appropriate. Improved clinical strategies, including assessment and treatment of opioid use disorder and multimodal pain management, may be needed for patients being considered for opioid discontinuation.

Acknowledgments: Our sincere appreciation to the following contributors: Greta Sweney, RPh, BCPS; Theresa O'Young, PharmD, $B C P S ;$ the many Adult Medicine Clinic providers who care for patients with chronic pain; and all the Adult Medicine Clinic patients whose lives have been affected by opioids.

Corresponding Author: Jocelyn R. James, MD; Department of Medicine, Division of General Internal Medicine, Harborview Medical Center, University of Washington School of Medicine, Seattle, WA, USA (e-mail:jorose@uw.edu).

Funding Information Chart review, data abstraction, and analytic support were provided by the University of Washington School of Medicine's Medical Student Research Training Program, the National Center for Advancing Translational Sciences of the National Institutes of Health under Award Number UL1 TROO2319, and the Division of General Internal Medicine at the University of Washington.

\section{Compliance with Ethical Standards:}

The study was approved by the Institutional Review Board at the University of Washington.

Conflict of Interest: The authors declare that they do not have a conflict of interest.

Disclaimer: No funding source had any role in study design, conduct, or reporting. This content is solely the responsibility of the authors and does not necessarily represent the official views of the National Institutes of Health.

\section{REFERENCES}

1. Daubresse M, Chang HY, Yu Y, et al. Ambulatory diagnosis and treatment of nonmalignant pain in the United States, 2000-2010. Med Care. 2013;51(10):870-878.

2. Warner M. Trends in Drug-poisoning Deaths Involving Opioid Analgesics and Heroin: United States, 1999-2012. Health E-Stats. 2014.
3. Okie S. A flood of opioids, a rising tide of deaths. $\mathrm{N}$ Engl $\mathrm{J}$ Med. 2010;363(21): 1981-1985.

4. Chou R DR, Devine B, Hansen R, Sullivan S, Jarvik JG, Blazina I, Dana T, Bougatsos C, Turner J. The Effectiveness and Risks of LongTerm Opioid Treatment of Chronic Pain. Evidence Report/Technology Assessment. (No 218).

5. Haffajee RL, Jena AB, Weiner SG. Mandatory use of prescription drug monitoring programs. Jama. 2015;313(9):891-892.

6. Dart RC, Surratt HL, Cicero TJ, et al. Trends in opioid analgesic abuse and mortality in the United States. N Engl J Med. 2015;372(3):241-248.

7. Group WSAMD, (AMDG). Interagency Guideline on Prescribing Opioids for Pain. 2015.

8. Seth P, Scholl L, Rudd RA, Bacon S. Overdose Deaths Involving Opioids, Cocaine, and Psychostimulants - United States, 2015-2016. MMWR Morb Mortal Wkly Rep 2018;67:349-358.

9. Hedegaard H, Warner M, Minino AM. Drug overdose deaths in the United States, 1999-2016. NCHS Data Brief. 2017;2016(No 294)

10. Group AMD. Opioid Dose Calculator.

11. Dunn KM, Saunders KW, Rutter CM, et al. Opioid prescriptions for chronic pain and overdose: a cohort study. Ann Intern Med. 2010;152(2):85-92.

12. Bohnert AS, Valenstein M, Bair MJ, et al. Association between opioid prescribing patterns and opioid overdose-related deaths. Jama. 2011;305(13):1315-1321.

13. Case A, Deaton A. Rising morbidity and mortality in midlife among white non-Hispanic Americans in the 21st century. Proc Natl Acad Sci U S A. 2015;112(49): 15078-15083.

14. Darnall BD, Ziadni MS, Stieg RL, Mackey IG, Kao MC, Flood P. Patient-Centered Prescription Opioid Tapering in Community Outpatients With Chronic Pain. JAMA Intern Med. 2018;178(5):707-708.

15. Dowell D, Haegerich TM, Chou R. CDC Guideline for Prescribing Opioids for Chronic Pain - United States, 2016. MMWR Recomm Rep. 2016;65(No. RR-1): 1-49.

16. Frank JW, Lovejoy TI, Becker wC, et al. Patient Outcomes in Dose Reduction or Discontinuation of Long-Term Opioid Therapy: A Systematic Review. Ann Intern Med. 2017;167(3):181-191.

17. Demidenko MI, Dobscha SK, Morasco BJ, Meath THA, Ilgen MA, Lovejoy TI. Suicidal ideation and suicidal self-directed violence following clinician-initiated prescription opioid discontinuation among long-term opioid users. Gen Hosp Psychiatry. 2017;47:29-35.

18. Hoffman J. Medicare is cracking down on opioids. Doctors fear pain patients will suffer. New York Times. 2018.

19. Darnall BD, Juurlink D, Kerns RD, et al. International Stakeholder Community of Pain Experts and Leaders Call for an Urgent Action on Forced Opioid Tapering. Pain Med. 2019;20(3):429-433.

20. Edlund MJ, Martin BC, Russo JE, DeVries A, Braden JB, Sullivan MD. The role of opioid prescription in incident opioid abuse and dependence among individuals with chronic noncancer pain: the role of opioid prescription. Clin J Pain. 2014;30(7):557-564.

21. Larochelle MR, Liebschutz JM, Zhang F, Ross-Degnan D, Wharam JF. Opioid Prescribing After Nonfatal Overdose and Association With Repeated Overdose: A Cohort Study. Ann Intern Med.. 2016;164(1):1-9.

22. Alford DP, LaBelle CT, Kretsch N, et al. Collaborative care of opioidaddicted patients in primary care using buprenorphine: five-year experience. Arch Intern Med. 2011;171(5):425-431.

Publisher's Note Springer Nature remains neutral with regard tojurisdictional claims in published maps and institutional affiliations. 